\title{
Insulin degludec is not associated with a delayed or diminished response to hypoglycaemia compared with insulin glargine in type 1 diabetes: a double-blind randomised crossover study
}

\author{
Gerd Koehler • Simon Heller • Stefan Korsatko • \\ Carsten Roepstorff • Søren Rasmussen • Hanne Haahr • \\ Thomas R. Pieber
}

Received: 14 May 2013 / Accepted: 21 August 2013/Published online: 22 September 2013

(C) The Author(s) 2013. This article is published with open access at Springerlink.com

\begin{abstract}
Aims/hypothesis Insulin degludec (Des(B30)LysB29( $\gamma$-Glu $\mathrm{N} \varepsilon$-hexadecandioyl) human insulin; IDeg) is a new basal insulin with an ultra-long flat action profile. The acute physiological responses to hypoglycaemia with IDeg and insulin glargine (A21Gly,B31Arg,B32Arg human insulin; IGlar) were compared.

Methods Twenty-eight adult type 1 diabetic patients with normal hypoglycaemia awareness (age $=41 \pm 12$ years, $\left.\mathrm{HbA}_{1 \mathrm{c}}=7.8 \pm 0.6 \%[62.8 \pm 7 \mathrm{mmol} / \mathrm{mol}]\right)$ were randomised to once-daily IDeg or IGlar for 5 days in a two-period crossover design. Participants and research staff were blinded to group assignment. Patients were assigned the lowest available randomisation number from a set of blinded randomisation codes provided by the trial sponsor. Hypoglycaemia was induced by administering three times the usual daily insulin dose at midnight on day 5. Plasma glucose (PG) was stabilised by glucose clamp $(5.5 \mathrm{mmol} / \mathrm{l})$ for $7-9 \mathrm{~h}$ post dosing. Next morning, PG was allowed to decrease stepwise from 5.5 to $3.5 \mathrm{mmol} / 1$ (maintained for $30 \mathrm{~min}$ ) to $2.5 \mathrm{mmol} / 1$ (for $15 \mathrm{~min}$ ). $\mathrm{PG}$ was then increased to $3.9 \mathrm{mmol} / \mathrm{l}$ (for $120 \mathrm{~min}$ ),
\end{abstract}

G. Koehler $\cdot$ S. Korsatko $\cdot$ T. R. Pieber $(\bowtie)$

Division of Endocrinology and Metabolism, Department of Internal Medicine, Medical University Graz,

Auenbruggerplatz 15, 8036 Graz, Austria

e-mail: endo@medunigraz.at

\section{S. Heller}

Academic Unit of Diabetes, Endocrinology and Metabolism, School of Medicine and Biomedical Sciences,

University of Sheffield, Sheffield, UK

C. Roepstorff $\cdot$ S. Rasmussen $\cdot$ H. Haahr

Novo Nordisk, Søborg, Denmark before being returned to baseline. Hypoglycaemic symptom score (HSS), hypoglycaemic awareness, cognitive function, counter-regulatory hormones and vital signs were assessed during each glucose plateau. The primary analysis was to compare IDeg vs IGlar with respect to HSS at nadir PG concentration $(2.5 \mathrm{mmol} / \mathrm{l})$.

Results The full analysis set for treatment comparisons comprised data from all 28 exposed patients. Rates of PG decline and PG at nadir were similar for IDeg and IGlar. No treatment differences in HSS (estimated difference: 0.17 [95\% CI -1.71 , 2.05]; $p>0.05$ ), cognitive function or awareness were observed at any time. Growth hormone and cortisol responses during hypoglycaemia were greater with IDeg than IGlar (AUC treatment ratio [IDeg/IGlar]: 2.44 [1.30, 4.60], $p<0.01$; and $1.23[1.01,1.50] ; p<0.05$ ), and adrenaline (epinephrine) responses trended higher $(1.40[0.96,2.04], p=0.07)$. The rates of recovery from hypoglycaemia were similar.

Conclusions/interpretation IDeg and IGlar elicit comparable symptomatic and cognitive responses to induced hypoglycaemia. IDeg may elicit a moderately greater endocrine response, but times to PG recovery were similar for the two insulins.

Trial registration ClinicalTrials.gov NCT01002768.

Funding Novo Nordisk.

Keywords Counter-regulation · Degludec · Glargine · Hormones $\cdot$ Hypoglycaemia $\cdot$ Type 1 diabetes
Abbreviations
CLIA Chemiluminescent immunoassay
HSS Hypoglycaemic symptom score
IDeg Insulin degludec
IGlar Insulin glargine
PG Plasma glucose 


\section{Introduction}

Insulin analogues are being developed to overcome the limitations of current insulin therapy by producing pharmacokinetic and pharmacodynamic profiles that more closely resemble physiological insulin release. The use of long-acting insulin analogues has been shown to reduce rates of hypoglycaemia, particularly at night and in individuals aiming for tight glycaemic control to minimise the risk of long-term complications. Despite this improvement, the annual prevalence of hypoglycaemia still remains between $30 \%$ and $40 \%$ in patients with type 1 diabetes. Nearly $50 \%$ of well-controlled individuals with longstanding type 1 diabetes experience at least one severe hypoglycaemic episode over 9-12 months [1]. Severe episodes of hypoglycaemia are less frequent in patients with diabetes who still have hypoglycaemia awareness [2], but these patients are still at significant risk of hypoglycaemia due to lack of adequate glucagon secretion and a reduced response of other counter-regulatory hormones [1]. Furthermore, with a longer duration of type 1 diabetes the risk for hypoglycaemia increases and hypoglycaemia awareness becomes reduced [3].

Although the risk of severe hypoglycaemia is partly related to impaired release of counter-regulatory hormones, the main clinical cause is inappropriately high insulin levels at vulnerable time points. Insulin degludec (Des(B30)LysB29( $\gamma$-Glu $\mathrm{N} \varepsilon$-hexadecandioyl) human insulin; IDeg) is a new ultralong-acting basal insulin analogue with an extended duration of action beyond $42 \mathrm{~h}$ [4], a flat insulin profile with once-daily dosing [5] and lower intrapatient variability [6]. Using IDeg, insulin-naive and insulin-treated patients with type 2 diabetes showed a significantly reduced risk of overall and nocturnal hypoglycaemia events [7]; in patients with type 1 diabetes, a similar result regarding nocturnal hypoglycaemia was found $[8,9]$. New insulin analogues with a very long action profile carry the potential risk of prolonged hypoglycaemia. This is particularly important in type 1 diabetes where the risk of hypoglycaemia is increased even further by poor hormonal counter-regulation and deteriorating hypoglycaemia awareness. Insulin analogues for type 1 diabetes therapy should thus be tested to ensure they are not associated with a further impairment of the remaining counter-regulation. To investigate the symptomatic, cognitive and counter-regulatory hormone response to hypoglycaemia induced by IDeg compared with that of an existing, clinically well-established, long-acting basal insulin, insulin glargine (A21Gly,B31Arg,B32Arg human insulin; IGlar), we performed a double-blind randomised crossover study in patients with type 1 diabetes.

\section{Methods}

Setting and participants We enrolled patients with type 1 diabetes between 18 and 65 years of age who were considered to still have hypoglycaemia awareness and were not suffering from any other comorbidities. Patients had a BMI between 18.0 and $28.0 \mathrm{~kg} / \mathrm{m}^{2}$, an $\mathrm{HbA}_{1 \mathrm{c}}$ between $6.7 \%(50 \mathrm{mmol} / \mathrm{mol})$ and $10.0 \%(86 \mathrm{mmol} / \mathrm{mol})$ and a total daily insulin dose below $1.2 \mathrm{U} / \mathrm{kg}$ (basal insulin $\geq 0.2 \mathrm{U} / \mathrm{kg}$ ). Key exclusion criteria were: prior IGlar therapy (within 3 months of screening); recurrent severe hypoglycaemia (more than one severe hypoglycaemic event during the last 12 months); hypoglycaemia unawareness (as judged by the medical investigator); pregnancy; impaired renal or hepatic function; impaired vision; and cardiac disease. All participants gave signed informed consent before participating in any trial activities. The trial was performed after approval by the local ethics committee and according to good clinical practice [10] and the Declaration of Helsinki [11].

Trial design This double-blind randomised crossover trial was designed to compare the development of symptomatic, cognitive and counter-regulatory hormone response to acute hypoglycaemia induced by IDeg or IGlar in adults with type 1 diabetes. Patients were randomly allocated to double-blind treatment with subcutaneous injections of either IDeg (Tresiba, $100 \mathrm{U} / \mathrm{ml} 3 \mathrm{ml}$ Penfill cartridges [Novo Nordisk, Bagsværd, Denmark]) or IGlar (Lantus, 100 U/ml, $3 \mathrm{ml}$ cartridges [Sanofi, Paris, France]) once daily for a runin period of 5 days over two separate treatment periods, with a wash-out period of 13-21 days between periods (during which usual insulin therapy was resumed). Patients were assigned the lowest available randomisation number from a set of blinded randomisation codes provided by the sponsor (Novo Nordisk).

A run-in period of 5 days was based on model calculations indicating that stable steady-state levels for both insulins were achieved within 2-3 days of once-daily dosing. Two days were added during which patients were at steady state, resulting in a total run-in period of 5 days. During the 5 day run-in period, each patient was asked to provide at least four plasma glucose (PG) measurements per day and to record any hypoglycaemic events. Patients were required to attend the clinic on each day for administration of the trial insulin. All injections were performed by a person not otherwise involved with the study to keep the double-blind character of the trial. Sequence allocation was carried out according to a computer-generated randomisation list. During the treatment periods, insulin aspart (B28Asp human insulin) (NovoRapid, $100 \mathrm{U} / \mathrm{ml}$ [Novo Nordisk]; available as a $3 \mathrm{ml}$ FlexPen) was administered as bolus insulin. During the first 4 days of each treatment period, the basal insulin dose was set to $80 \%$ of the patients' total daily basal insulin dose to minimise the risk of hypoglycaemia, and dose levels remained the same during both treatment periods. Insulin was administered s.c. in the thigh at approximately 20:00 hours on days $1-4$ in each treatment period. 
On day 5 the stepwise hypoglycaemic glucose clamp procedure was started. Patients were admitted to the clinic before 20:00 hours for an in-house stay of at least $50 \mathrm{~h}$. At the discretion of the investigator, patients stayed at the clinic to ensure stabilisation of blood glucose values after completion of glucose clamp procedures.

Stepwise hypoglycaemic glucose clamp procedure We were able to initiate the hypoglycaemic glucose clamp procedure as planned in all patients as no patients had experienced any hypoglycaemic episodes ( $\mathrm{PG} \leq 3.9 \mathrm{mmol} / \mathrm{l}$ ) during the previous $24 \mathrm{~h}$. To achieve a PG target of $5.5 \mathrm{mmol} / \mathrm{l}( \pm 30 \%)$ for the runin period of 7-9 $\mathrm{h}$, patients received a variable intravenous glucose infusion (10\%; Braun Infusomat FM; Melsungen, Germany) or human soluble insulin infusion (40 U Actrapid [100 U/ml] in $99.6 \mathrm{ml} \mathrm{NaCl}[154 \mathrm{mmol} / \mathrm{l}]$ ) which was initiated at approximately 22:00 hours. To facilitate hypoglycaemia induction, a three-times-higher basal insulin dose was administered at midnight (range of timing $\pm 3 \mathrm{~min}$ ), replacing the regular basal insulin dose given at 20:00 hours (Fig. 1). After the midnight dosing, insulin infusion was stopped when glucose levels had dropped by approximately $0.3 \mathrm{mmol} / \mathrm{l}$ $(5 \mathrm{mg} / \mathrm{dl}$ ). At $30 \mathrm{~min}$ prior to hypoglycaemia induction (in the morning), $\mathrm{PG}$ was maintained at $5.5 \mathrm{mmol} / \mathrm{l}$ but with a deviation of only $\pm 10 \%$. Patients remained fasting in a supine or semi-supine position throughout the hypoglycaemic glucose clamp procedure, except when cognitive function tests were performed. Glucose infusion was stopped to induce hypoglycaemia. PG was allowed to decrease to target levels of 3.5 and $2.5 \mathrm{mmol} / 1$ (nadir) in a stepwise fashion. Variable glucose infusion was resumed at each step to maintain PG at the target level for fixed periods of 30 and $15 \mathrm{~min}$, respectively. Controlled recovery from hypoglycaemia was initiated after the nadir period. Glucose was infused at a fixed rate of $5.5 \mathrm{mg} \mathrm{kg}^{-1} \mathrm{~min}^{-1}$ until PG reached the recovery target of $3.9 \mathrm{mmol} / \mathrm{l}$. PG was clamped at $3.9 \mathrm{mmol} / \mathrm{l}$ by variable i.v. glucose infusion for $120 \mathrm{~min}$, before being increased to $5.5 \mathrm{mmol} / \mathrm{l}$, where it was clamped again until it was deemed safe to terminate the clamp procedure.

Arterialised whole-blood samples were drawn for PG, counter-regulatory hormones and pharmacokinetic insulin analysis throughout the procedure. Moreover, hypoglycaemic symptoms, hypoglycaemic awareness, vital signs and cognitive function were measured at time points corresponding to specific PG concentrations according to Fig. 1.

Symptoms of hypoglycaemia and hypoglycaemic awareness Symptoms of hypoglycaemia at each PG target level (Fig. 1) were assessed using a validated questionnaire (Edinburgh Hypoglycaemia Scale [12]). This scale classifies hypoglycaemic symptoms into autonomic symptoms (sweating, palpitations, shaking and hunger), neuroglycopenic symptoms (confusion, drowsiness, odd behaviour, speech difficulty and incoordination) and non-specific symptoms (headache and nausea) [12, 13]. Each of the 11 symptoms was scored by the patient on a seven point interval scale ranging from 1 (= not at all) to 7 (= a great deal). The total score at each time point was calculated as the mean score for all symptoms multiplied by 11 .
Fig. 1 Stepwise glucose clamp procedure. Run in: participants were clamped at a PG level of $5.5 \mathrm{mmol} / \mathrm{l}(100 \mathrm{mg} / \mathrm{dl}) 2 \mathrm{~h}$ before dosing until 7-9 $\mathrm{h}$ after dosing. Hypoglycaemia induction: variable i.v. glucose was terminated and PG was allowed to decrease to $3.5 \mathrm{mmol} / \mathrm{l}(63 \mathrm{mg} / \mathrm{dl})$, maintained for $30 \mathrm{~min}$, and thereafter to a target nadir of $2.5 \mathrm{mmol} / \mathrm{l}(45 \mathrm{mg} / \mathrm{dl})$, maintained for $15 \mathrm{~min}$. Recovery: at end of nadir PG, a fixed i.v. glucose infusion $\left(5.5 \mathrm{mg} \mathrm{kg}^{-1} \mathrm{~min}^{-1}\right)$ was initiated, and PG was allowed to increase to $3.9 \mathrm{mmol} / \mathrm{l}(70 \mathrm{mg} / \mathrm{dl})$, maintained for $120 \mathrm{~min}$, and thereafter to $5.5 \mathrm{mmol} / \mathrm{l}(100 \mathrm{mg} / \mathrm{dl})$. Thick broken line, variable glucose infusion rate (GIR); thick unbroken line, fixed i.v. glucose infusion $\left(5.5 \mathrm{mg} \mathrm{kg}^{-1} \mathrm{~min}^{-1}\right)$

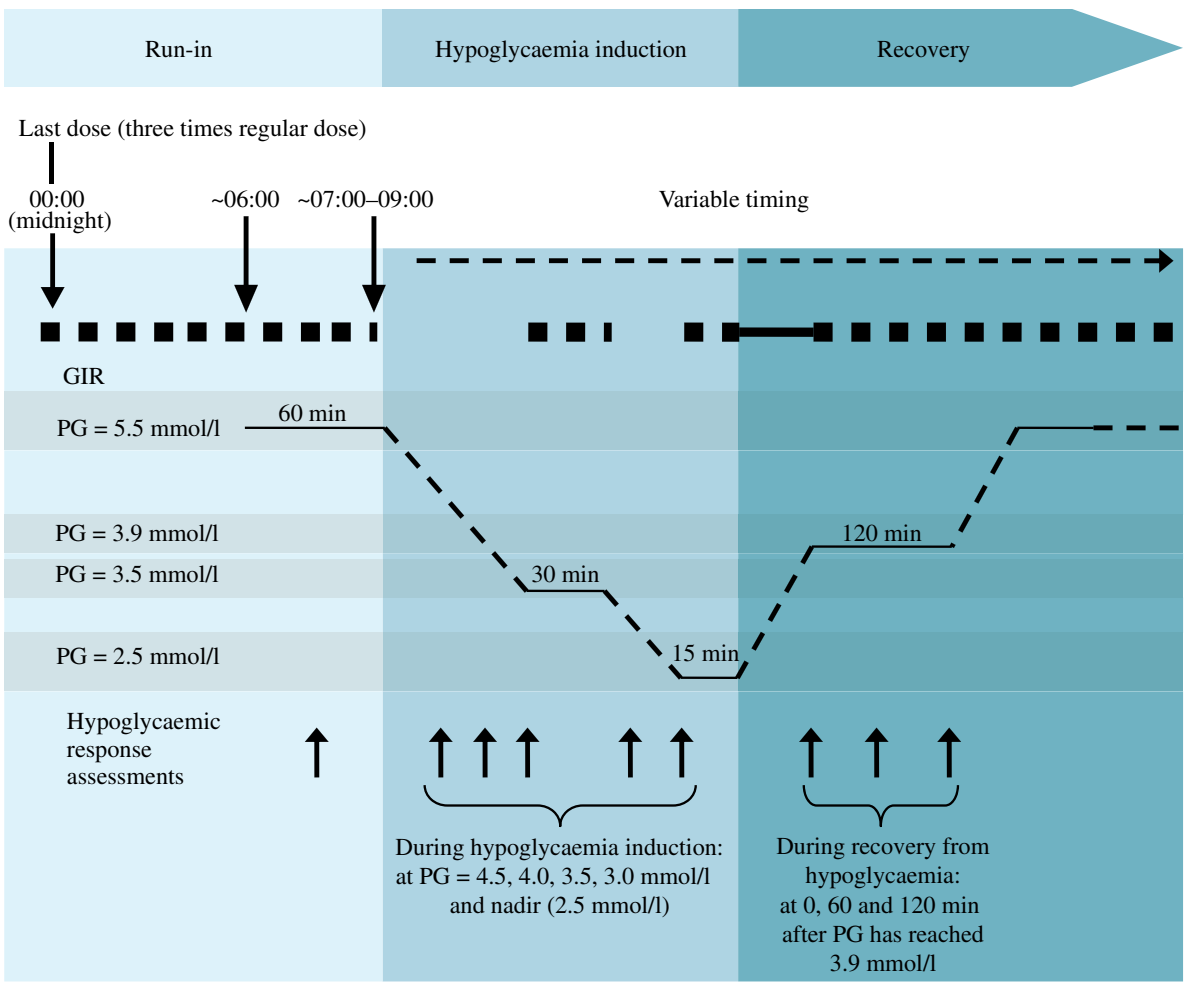


Hypoglycaemic awareness was assessed by asking the patient the question 'Do you feel a hypo?'

Cognitive function Cognitive function at each $\mathrm{PG}$ target level (Fig. 1) was evaluated by using three cognitive function tests recognised as being sensitive to hypoglycaemia [14]: trail making B [15, 16]; digit symbol substitution test [17]; and the four-choice reaction time [18].

Counter-regulatory hormones Counter-regulatory hormone responses to hypoglycaemia were measured at each PG target level (Fig. 1). Plasma concentrations of adrenaline and noradrenaline (norepinephrine) were quantified by RIA (DRG Diagnostics, Springfield, IL, USA) and glucagon by RIA (ICN, New York, NY, USA). Serum concentration of growth hormone was measured using a chemiluminescent immunoassay (CLIA) (Immolite; Siemens Healthcare Diagnostics, New York, NY, USA) and cortisol by CLIA (Centaur; Siemens Healthcare Diagnostics).

Vital signs Vital signs (diastolic and systolic blood pressure and pulse) at each PG target level (Fig. 1) were assessed after the participant had rested for at least $5 \mathrm{~min}$ in a supine position.

Statistical analysis For the primary endpoint, hypoglycaemic symptom score (HSS) at nadir, using an SD of 13.5 [19] and a significance level of $5 \%$, it was calculated that 26 completers were required to yield sufficient power $(83 \%)$ to detect an eight point difference between IDeg and IGlar. Twenty-eight patients were enrolled to allow for dropouts.

Endpoints were compared between IDeg and IGlar using a linear mixed model, with treatment period and treatment as fixed effects and patients as a random effect (assuming the random patient effect and error term were independent). For analysis of baseline-adjusted HSS, baseline HSS was included as a continuous covariate. For PG concentration at nadir and counter-regulatory hormone response during development of hypoglycaemia, estimated as AUC for the period between PG $4.0 \mathrm{mmol} / \mathrm{l}$ and PG nadir, endpoints were log-transformed before analysis.

Rates of increase in HSS and vital signs and deterioration in cognitive function during hypoglycaemia induction were analysed by comparing the slopes for IDeg and IGlar, including target PG level as a continuous covariate (4.5, 4.0, 3.5, 3.0 and $2.5 \mathrm{mmol} / 1$ [nadir]) in the model, an interaction between treatment and target PG level, and adjusted for baseline value. The same approach was used for rate of increase in counter-regulatory hormone response (though log-transformed and not baseline adjusted). The assumption of a common intercept for IDeg and IGlar at PG level $5.5 \mathrm{mmol} / \mathrm{l}$ was tested with a significance level of $5 \%$ for each endpoint.
PG decline with IDeg and IGlar was compared by calculating the time from the start of hypoglycaemic induction (5.5 mmol/l) until each PG target level $(4.5,4.0,3.5$, $3.0 \mathrm{mmol} / 1$ and nadir). Rate of PG decline was evaluated from target $P G=4.5 \mathrm{mmol} / 1$ to nadir to demonstrate that the profiles were comparable during development of hypoglycaemia. Differences in time were log-transformed and analysed using the same general model as described above, though with target PG level as a continuous covariate and an interaction between treatment and target PG levels (assuming the possibility of different treatment slopes and a common intercept for IDeg and IGlar at $P G=5.5 \mathrm{mmol} / \mathrm{l}$ ).

During recovery from hypoglycaemia, the slopes for IDeg and IGlar were compared for each variable using the same models as described for development of hypoglycaemia, substituting target PG level with time (as a continuous covariate) at 0,60 and $120 \mathrm{~min}$.

The amount of glucose required to stabilise individual glucose levels at baseline $(5.5 \mathrm{mmol} / \mathrm{l})$, during development of hypoglycaemia ( 3.5 and $2.5 \mathrm{mmol} / \mathrm{l}$ [nadir]) and during the entire recovery period (from end of nadir PG to end of the $2 \mathrm{~h}$ period at $3.9 \mathrm{mmol} / \mathrm{l}$ ) was estimated as AUC of glucose infusion rate, log-transformed and analysed using the general model.

\section{Results}

Participant characteristics and trial flow A total of 28 eligible men and women with type 1 diabetes were enrolled in the trial (Fig. 2). Baseline demographic characteristics are summarised in Table 1. Two patients were withdrawn

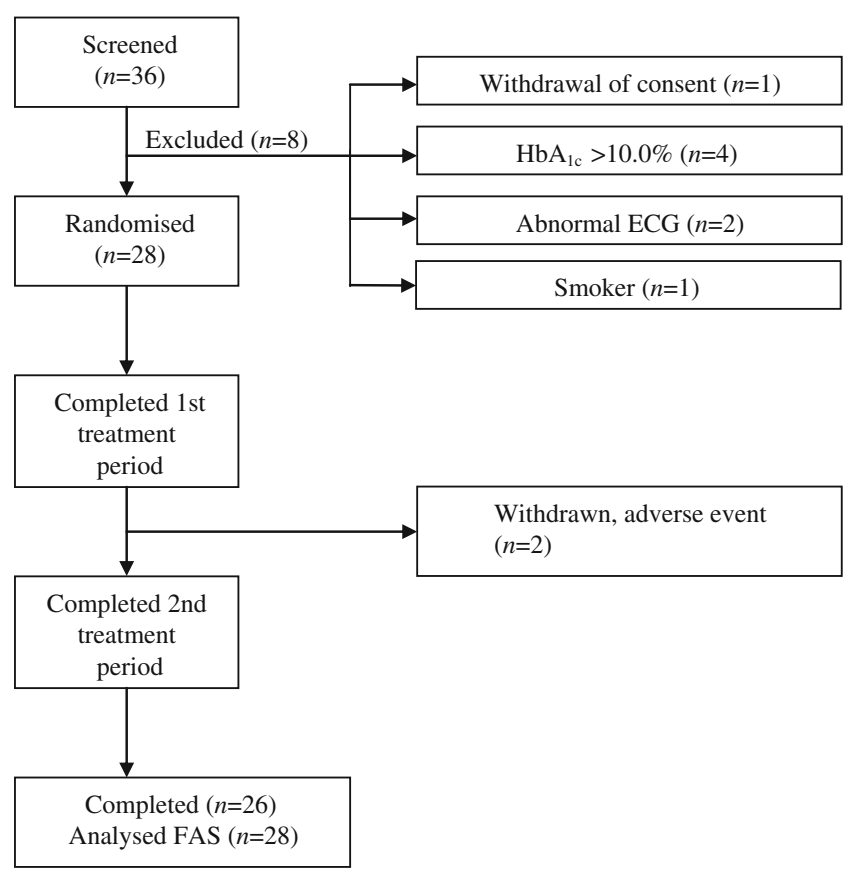

Fig. 2 Flow of participants through the trial. FAS, full analysis set 
Table 1 Demographic and baseline characteristics $(n=28)$

\begin{tabular}{lll}
\hline Characteristic & Mean $\pm \mathrm{SD}$ & Range \\
\hline Sex (female/male) & $8 / 20$ & \\
Age (years) & $40.7 \pm 11.7$ & $22.0-65.0$ \\
$\mathrm{BMI}\left(\mathrm{kg} / \mathrm{m}^{2}\right)$ & $25.1 \pm 2.7$ & $19.1-27.9$ \\
Duration of diabetes (years) & $20.6 \pm 9.9$ & $4.5-50.5$ \\
$\mathrm{HbA}_{1 \mathrm{c}}(\%)$ & $7.8 \pm 0.6$ & $6.9-9.3$ \\
$\mathrm{HbA}_{1 \mathrm{c}}(\mathrm{mmol} / \mathrm{mol})$ & $62.8 \pm 7$ & $52-78$ \\
Fasting PG (mmol/l) & $9.5 \pm 3.4$ & $3.7-16.9$ \\
Basal insulin $\left(\mathrm{U} \mathrm{kg}{ }^{-1}\right.$ day $\left.^{-1}\right)$ & $0.32 \pm 0.1$ & $0.20-0.59$ \\
\hline
\end{tabular}

after the first treatment period because of the onset of adverse events. The full analysis set for treatment comparisons comprises data from all 28 exposed patients.

Glycaemic state prior to the hypoglycaemic clamp experiments On each of the 5 days prior to the hypoglycaemic clamp experiment, glycaemic control was similar for IDeg and IGlar, measured by four-point self-measured PG profiles. For both insulins, low and similar numbers of hypoglycaemic episodes were reported on each of the 5 days prior to the hypoglycaemic clamp experiment (data not shown). The trial protocol required that no hypoglycaemia occurred for any patient in the $24 \mathrm{~h}$ immediately before the start of the clamp experiment.

Plasma glucose and glucose infusion rate As required by the study design, hypoglycaemia induction could only commence once PG had been at $5.5 \mathrm{mmol} / 1 \pm 10 \%$ for $30 \mathrm{~min}$. During this period, geometric mean PG levels (CV\%) were $5.5 \mathrm{mmol} / \mathrm{l}(3.8 \%)$ and $5.5 \mathrm{mmol} / \mathrm{l}(4.9 \%)$ for IDeg and IGlar, respectively. Hypoglycaemia induction could occur between 07:00 and 09:00 hours in the morning of day 5; mean actual time of hypoglycaemia induction was 07:26 hours for IDeg and 07:32 hours for IGlar.

Mean PG profiles were similar with IDeg and IGlar throughout the hypoglycaemic clamp procedure (Fig. 3a). The rate of PG decline from baseline $(5.5 \mathrm{mmol} / \mathrm{l})$ to $4.5 \mathrm{mmol} / \mathrm{l}$ was slightly lower with IDeg than IGlar, but rates were identical for both insulins at PG below $4.5 \mathrm{mmol} / \mathrm{l}$. There was no significant difference in nadir PG levels between treatments (estimated geometric mean [CV\%] 2.6 [9.4] vs 2.5 [9.7] mmol/1; estimated treatment ratio [IDeg/IGlar]: 1.03 [95\% CI $0.99,1.08], p=0.12$ ). Similarly, time to PG recovery from nadir to $3.9 \mathrm{mmol} / \mathrm{l}$ (when glucose was infused i.v. at a fixed rate of $5.5 \mathrm{mg} \mathrm{kg}^{-1} \mathrm{~min}^{-1}$; see Fig. 1) was not significantly different for the two treatments: estimated geometric mean 8.1 vs $10.9 \mathrm{~min}$; and estimated treatment ratio $0.75(95 \%$ CI $0.56,1.02), p=0.07$.

Variable glucose infusion rates during the clamp procedure tended to be lower with IDeg than IGlar (Fig. 3b). Significant
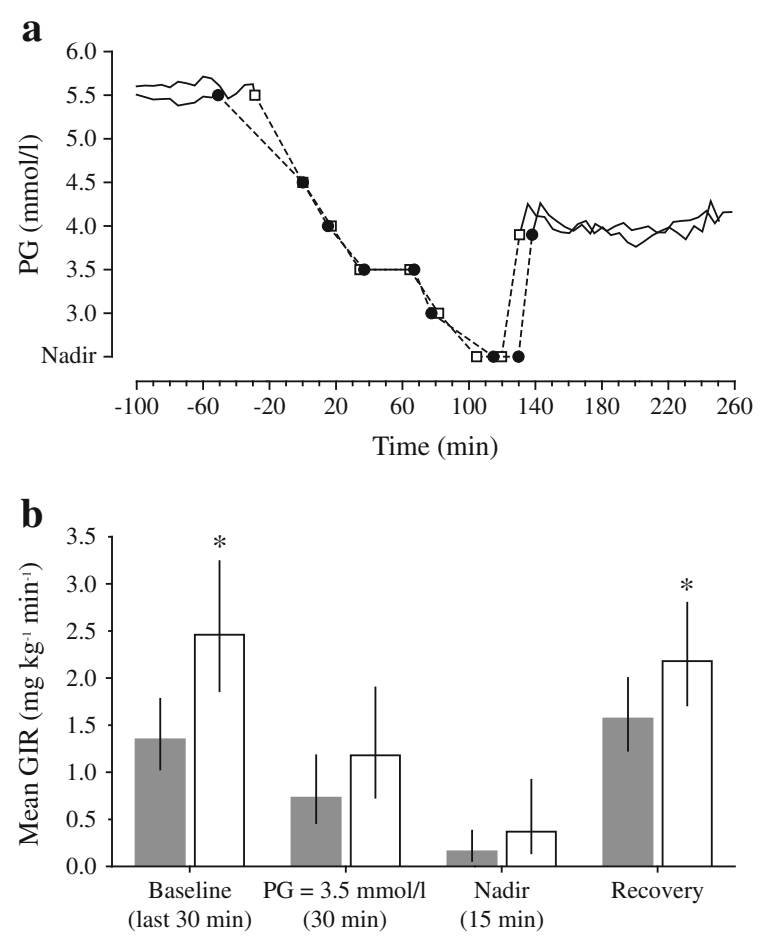

Fig. 3 Mean time to PG targets (a) and mean glucose infusion rate (b) during development of, and recovery from, hypoglycaemia. In (a), time point zero has been defined as when $P G=4.5 \mathrm{mmol} / 1$, because a decline in PG below $4.5 \mathrm{mmol} / \mathrm{l}$ is considered to be the threshold for hypoglycaemic response. Black circles, IDeg; white squares, IGlar. In (b), *denotes statistically significant estimated treatment difference in rate of glucose infusion $(p<0.05)$. Grey bars, IDeg; white bars, IGlar; narrow vertical black bars, $95 \%$ CI. GIR, glucose infusion rate

treatment differences in the rate of glucose infusion were observed both at baseline (PG target $=5.5 \mathrm{mmol} / \mathrm{l})$ (estimated treatment ratio [IDeg/IGlar]: 0.55 [95\% CI 0.41, 0.74], $p<0.01$ ) and during the post nadir $2 \mathrm{~h}$ clamp $(3.9 \mathrm{mmol} / \mathrm{l})$ (estimated treatment ratio: 0.71 [95\% CI 0.53, 0.93], $p=0.02$ ).

HSS Mean HSS increased similarly for IDeg and IGlar during hypoglycaemia induction (Fig. 4). HSS increased for both insulins when PG level dropped below $4.0 \mathrm{mmol} / \mathrm{l}$. There was no significant difference in baseline-adjusted HSS at nadir (the primary endpoint) between IDeg and IGlar (5.00 vs 4.83, estimated treatment difference 0.17 [95\% CI $-1.71,2.05]$ ). During recovery from hypoglycaemia, mean HSS returned to baseline at a similar rate for IDeg and IGlar (Fig. 4).

Cognitive function scores Overall deterioration and restoration of cognitive function during development of and recovery from hypoglycaemia was similar with IDeg and IGlar (Fig. 5). Measurable treatment differences were detected only during the development of hypoglycaemia, where the number of correct responses given to the digit symbol substitution test (DSST; Fig. 5b) declined at a significantly greater rate with IGlar than with IDeg, and the treatment difference in DSST at 


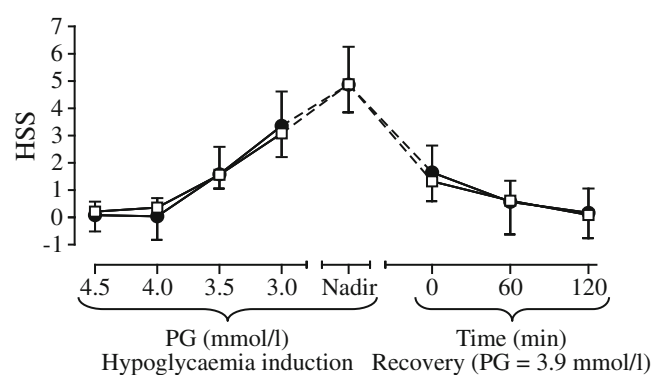

Fig. 4 Baseline-adjusted HSS during development of, and recovery from, hypoglycaemia. Black circles, IDeg; white squares, IGlar; vertical bars, SEM

PG nadir was significant (estimated treatment difference in slope (IDeg-IGlar) 5.93 [95\% CI 1.23, 10.62], $p<0.05$ ).

Hypoglycaemic awareness Hypoglycaemic awareness was similar at all PG levels for IDeg and IGlar, occurring in 8\% and $4 \%$ of patients, respectively, at $5.5 \mathrm{mmol} / \mathrm{l}$, increasing to a maximum of $74 \%$ and $68 \%$ of patients at $P G$ nadir (not tested).

Counter-regulatory hormones Counter-regulatory hormone responses during development of hypoglycaemia were moderately greater with IDeg than with IGlar for three of the five hormones monitored (Fig. 6). No differences in hormone levels were detected between treatments at baseline $(5.5 \mathrm{mmol} / 1, p>0.05)$. Growth hormone, cortisol and adrenaline levels increased more rapidly during development of hypoglycaemia after treatment with IDeg than with IGlar (estimated treatment ratio in slope [IDeg/IGlar] 1.35 [95\% CI 1.19, 1.54], 1.03 [95\% CI 1.00, 1.06] and 1.07 [95\% CI 1.01, 1.14], respectively). Overall growth hormone and cortisol responses during development of hypoglycaemia were significantly greater with IDeg than with IGlar (growth hormone AUC [ng min $\mathrm{ml}^{-1}$ ] estimated geometric mean 498 vs 204, estimated treatment ratio 2.44 [95\% CI 1.30, 4.60], $p<0.01$; and cortisol AUC [nmol min $1^{-1}$ ] 29,891 vs 24,301 , treatment ratio 1.23 [95\% CI 1.01 , $1.50], p<0.05)$. These differences were also significant at nadir PG, with IDeg being greater than IGlar (estimated treatment ratio $1.85[95 \% \mathrm{CI} 1.06,3.21], p=0.031$, and 1.16 [95\% CI 1.00, 1.34], $p=0.049$ for growth hormone and cortisol response, respectively). A similar though nonsignificant trend was observed for adrenaline response during development of hypoglycaemia (AUC [pmol min $1^{-1}$ ] estimated geometric mean 36,233 vs 25,804 , estimated treatment ratio 1.40 [95\% CI 0.96, 2.04], $p=0.07$ ); however, no significant difference in plasma adrenaline levels could be detected between IDeg and IGlar at nadir. Noradrenaline and glucagon levels developed similarly during acute hypoglycaemia when induced by either insulin. Counter-regulatory hormones returned to baseline at similar rates for IDeg and IGlar. a
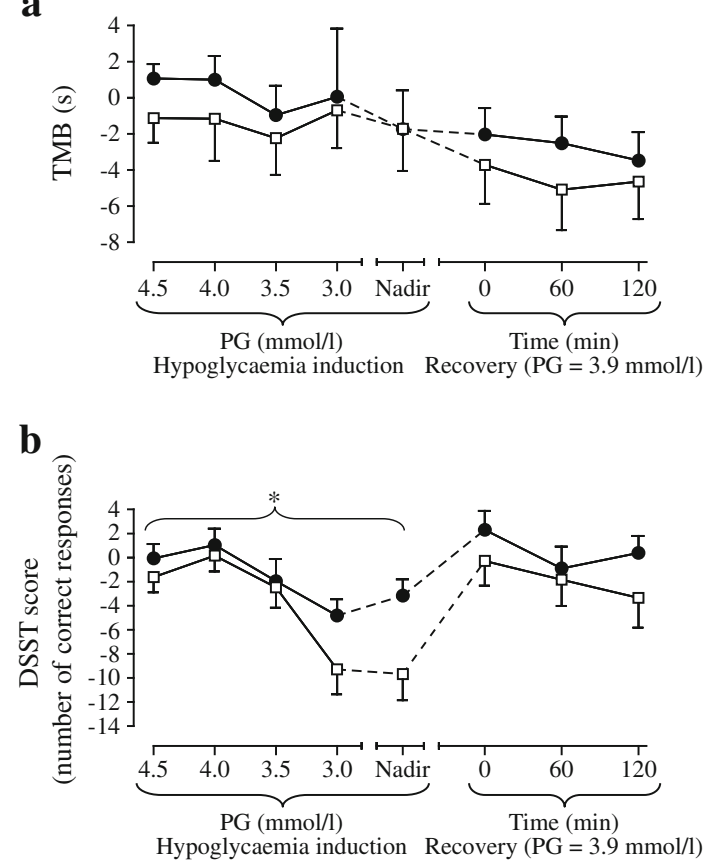

c

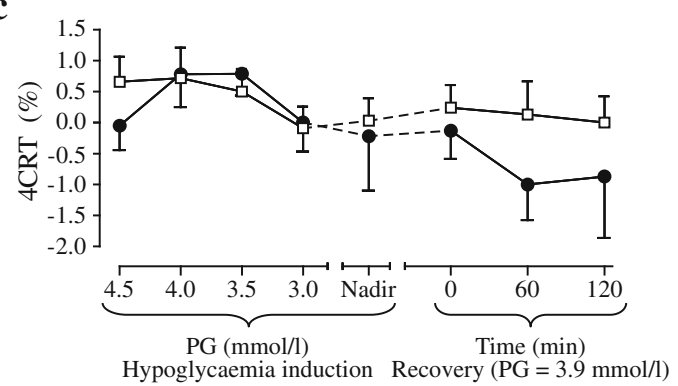

d

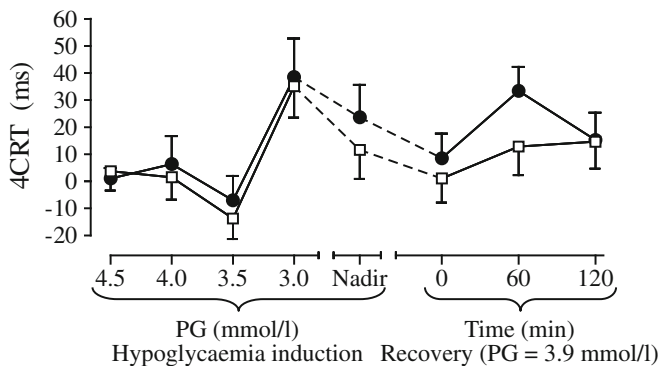

Fig. 5 Baseline-adjusted cognitive function performance test scores during development of, and recovery from, hypoglycaemia for (a) trail making $\mathrm{B}$, (b) digit symbol substitution test (DSST), (c) four-choice reaction time (percentage correct answers) and (d) four-choice reaction time (response time). Black circles, IDeg; white squares, IGlar; vertical bars, SEM. *denotes statistically significant estimated treatment difference in slopes $(p<0.05)$. TMB, trail making B; 4CRT, four-choice reaction time

Vital signs Diastolic blood pressure decreased similarly during the development of hypoglycaemia with both treatments: comparing IDeg with IGlar (estimated treatment difference in slope [IDeg-IGlar] $-0.42[95 \%$ CI -1.18 , $0.35])$. No effect on either systolic blood pressure or pulse was observed with either IDeg or IGlar. At nadir PG, there 

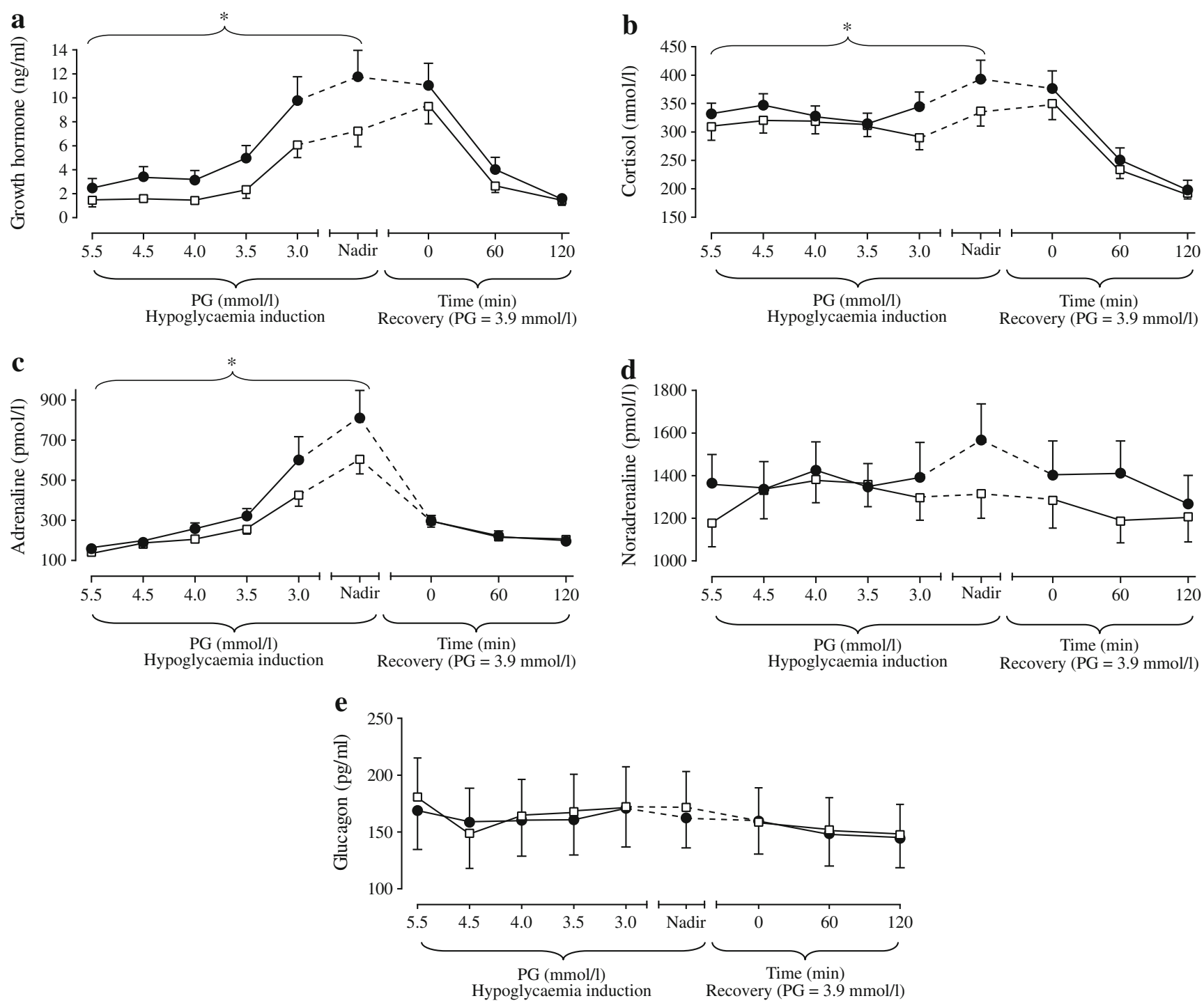

Fig. 6 Counter-regulatory hormone response during development of, and recovery from, hypoglycaemia for (a) growth hormone, (b) cortisol, (c) adrenaline, (d) noradrenaline and (e) glucagon. Black

was no statistically significant difference between IDeg and IGlar with regard to pulse or blood pressure. Vital signs returned to baseline at similar rates for IDeg and IGlar.

Safety and tolerability One serious adverse event was reported after the first dose in the IDeg treatment period. At 30 min after trial drug injection, one patient developed a mild swelling of the face and a general erythema of the skin (reported as an anaphylactic reaction). The patient was subsequently withdrawn from the trial and recovered completely without the need for medical intervention. Another patient withdrew after completing the IDeg treatment period because of the onset of mild influenza. Overall, 28 adverse events were reported for 18 patients; the majority of events were mild headache (16 events, seven with IDeg and nine with IGlar). Two adverse events (myopia and circles, IDeg; white squares, IGlar; vertical bars, SEM. *denotes statistically significant estimated treatment difference in slopes $(p<0.05)$

anaphylactic reaction) were considered to have a possible relation to IDeg. No clinically significant changes in vital signs, ECG or safety laboratory variables from screening to follow-up were observed.

\section{Discussion}

This is the first study to examine how the ultra-long-acting basal insulin IDeg (estimated duration of action $>42 \mathrm{~h}$ ) affects the hypoglycaemic response during development of, and recovery from, hypoglycaemia in patients with type 1 diabetes. The results indicate that there are no marked differences in hypoglycaemic response or hypoglycaemic awareness induced by IDeg compared with the long-acting basal insulin IGlar. Similar effects on hypoglycaemic symptom and cognitive 
function scores during hypoglycaemia induction, PG nadir and recovery from hypoglycaemia were observed with both insulins. One interesting observation was a modest effect in enhancing some of the counter-regulatory hormone responses around the glucose nadir with IDeg, and a lower glucose infusion rate during recovery with IDeg compared with IGlar; however, this had no obvious effect on the HSS or cognitive function.

The main strengths of this trial relative to other hypoglycaemic experimental designs include the extensive measurement of symptomatic, cognitive and counter-regulatory hormonal responses and the double-blind crossover design, which eliminates potential investigator and patient bias as well as inter-patient variability. Given that IDeg has a duration of action $>42 \mathrm{~h}$, we considered it important to characterise how recovery from hypoglycaemia induced by IDeg develops with respect to the increase in PG and the persistence of symptoms and effects on cognitive function in advance of the introduction of IDeg into clinical practice. Finally, we included people with type 1 diabetes, rather than healthy patients, to ensure the pharmacodynamic response was not confounded by endogenous insulin production [20-22]. In addition, intrapatient variability is likely to be lower in type 1 diabetes because of the lack of substantial endogenous insulin production. Though we recognise that a degree of hypoglycaemia unawareness is inevitable in a study population with an average diabetes duration of 21 years and is a study limitation, our main goal was to assess the counter-regulation in a clinically relevant type 1 diabetes population. Results, particularly from HSS, indicate sufficient hypoglycaemia awareness in all participating patients. Patients with severe hypoglycaemia unawareness were excluded from the study altogether.

We used a stepwise hypoglycaemic clamp technique using s.c. delivery in an attempt to achieve highly standardised study conditions to compare physiological and counter-regulatory responses with IDeg and IGlar. Although experimental hypoglycaemic studies involving other basal insulins have generally used a model incorporating i.v. delivery [23-25], we considered s.c. administration more appropriate. This approach is more clinically relevant, particularly as the mode of protraction for both IDeg and IGlar is activated by s.c. administration. We attempted to standardise trial conditions by administering IDeg and IGlar at midnight, approximately $7 \mathrm{~h}$ prior to inducing hypoglycaemia. A dose three times higher than the patient's regular insulin dose was administered on day 5 to try to achieve an appropriately low glucose target. The design was based on pharmacokinetic and pharmacodynamic modelling of existing data for both insulins. Thus, we set out to control for differing glycaemia, and to achieve comparability despite the use of two insulins with different action profiles. The delay in initiating hypoglycaemia (by maintaining patients in a euglycaemic clamp) until 7-9 h after administration of trial insulin was designed to control for differences in depth and duration of hypoglycaemia, and to control for the fall in glucose towards the hypoglycaemic plateau. However, variable glucose infusion rates during the clamp procedure tended to be lower with IDeg than IGlar, particularly at baseline (7-9 $\mathrm{h}$ post dosing) and during the recovery phase. Therefore, we cannot draw any firm conclusions regarding the relation between the observed differences in counter-regulatory hormone responses and in particular the rate of glucose recovery.

While the intensity of the hypoglycaemic response is multifactorial, important determinants are probably prior glycaemic experience, absolute PG level and the rate of PG decline. The comparable self-measured PG profiles and number of hypoglycaemic episodes suggest that glucose control was similar for IDeg and IGlar during the 5 days leading up to the hypoglycaemic clamp experiment. Moreover, in this trial, mean PG was similar with IDeg and IGlar throughout the hypoglycaemic clamp procedure. The rate of decline during hypoglycaemic induction was identical for both insulins once PG fell below $4.5 \mathrm{mmol} / \mathrm{l}$, suggesting that the hypoglycaemic stimulus was comparable during clamp procedures. This is an important criterion for comparing the response between the two insulins.

We observed moderate differences in counter-regulatory hormone responses (as measured by the rate of increase, AUC and hormone level at PG nadir) during the development of hypoglycaemia for three of the five counter-regulatory hormones (adrenaline, growth hormone and cortisol) when induced with IDeg compared with IGlar; however, the underlying mechanism for the observed differences could not be identified in this study. As glucagon responses to hypoglycaemia are attenuated by both type 1 diabetes and hyperinsulinaemia [21], it is not surprising that no differences in glucagon response were observed. Elevated cortisol levels have been associated with autonomic failure [26] but more recent studies could not confirm this finding [27, 28]. Enhanced counterregulatory hormone response might contribute to a reduced risk of hypoglycaemia, an important limiting factor in achieving good glycaemic control with intensified insulin therapy.

Although basal insulins such as IGlar are associated with a reduction in severe and nocturnal hypoglycaemia compared with neutral protamine Hagedorn insulin [29, 30], this appears to be due to improvements in insulin kinetics rather than to differences in glucose counter-regulation. Comparable physiological responses to induced hypoglycaemia have been shown for IGlar and human insulin in a stepped hypoglycaemic clamp [24] and clinical trials have shown comparable glycaemic control with IDeg and IGlar when treated to target [31, 32]. Moreover, results from the large-scale confirmatory clinical trials provide promising evidence of a lower risk of hypoglycaemia with IDeg compared with IGlar, particularly at night [7-9].

In conclusion, this trial provides reassuring data regarding the response to hypoglycaemia association with IDeg. 
IDeg and IGlar elicit comparable symptomatic and cognitive responses to induced hypoglycaemia, and recovery from hypoglycaemia remains similar. Furthermore, our trial suggests that IDeg may elicit a more pronounced endocrine response to hypoglycaemia than IGlar, albeit modest. Whether this translates into a clinically relevant difference cannot be answered by our trial. Adequately powered randomised multicentre studies in clinical settings are needed to establish the relevance of the effects of this new insulin on hypoglycaemia awareness, glucose counter-regulation and particularly rates of severe hypoglycaemia among individuals with type 1 diabetes.

Acknowledgements We thank the investigators, trial staff and participants for their participation. The authors wish to thank Mark White, Paul Drake (both Novo Nordisk) and Selma Mautner (Medical University of Graz) for editorial assistance.

\section{Funding This trial was funded by Novo Nordisk.}

Duality of interest Gerd Koehler has received lecture fees from Novo Nordisk, AstraZeneca, Bristol-Myers Squibb and Eli Lilly. Simon Heller has received: consulting fees from Abbott, Amylin, AstraZeneca, Eli Lilly, Johnson and Johnson, Mannkind, Novo Nordisk, Sanofi and Takeda; lecture fees from Amylin, AstraZeneca, Aventis, Eli Lilly, Johnson and Johnson, Mannkind, Merck, Novo Nordisk, Sanofi, Takeda and Servier; and research support from Amylin, AstraZeneca, Sanofi, Eli Lilly, Johnson and Johnson, Mannkind, Novo Nordisk and Takeda. Carsten Roepstorff, Søren Rasmussen and Hanne Haahr are employees of the sponsor (Novo Nordisk) and own stock in the company. Thomas R. Pieber has participated in advisory panels and acted as a consultant for Novo Nordisk. Stefan Korsatko declares that there is no duality of interest associated with his contribution to this manuscript.

Contribution statement All authors confirm that they meet the International Committee of Medical Journal Editors (ICMJE) uniform requirements for authorship and that they have contributed to: the conception and design, or analysis and interpretation of data; and drafting of the article or revising it critically for important intellectual content. All authors have approved the final version of the manuscript to be published.

Open Access This article is distributed under the terms of the Creative Commons Attribution License which permits any use, distribution, and reproduction in any medium, provided the original author(s) and the source are credited.

\section{References}

1. UK Hypoglycaemia Study Group (2007) Risk of hypoglycaemia in types 1 and 2 diabetes: effects of treatment modalities and their duration. Diabetologia 50:1140-1147

2. Gold AE, MacLeod KM, Frier BM (1994) Frequency of severe hypoglycemia in patients with type I diabetes with impaired awareness of hypoglycemia. Diabetes Care 17:697-703

3. McCrimmon RJ, Sherwin RS (2010) Hypoglycemia in type 1 diabetes. Diabetes 59:2333-2339

4. Jonassen I, Havelund S, Hoeg-Jensen T et al (2012) Design of the novel protraction mechanism of insulin degludec, an ultra-longacting basal insulin. Pharm Res 29:2104-2114
5. Gough SCL, Harris S, Woo V, Davies M (2013) Insulin degludec: overview of a novel ultra long-acting basal insulin. Diabetes Obes Metab 15:301-309

6. Heise T, Hermanski L, Nosek L et al (2012) Insulin degludec: four times lower pharmacodynamic variability than insulin glargine under steady-state conditions in type 1 diabetes. Diabetes Obes Metab 14:859-864

7. Garber AJ, King AB, del Prato S et al (2012) Insulin degludec, an ultra-longacting basal insulin, versus insulin glargine in basalbolus treatment with mealtime insulin aspart in type 2 diabetes (BEGIN Basal-Bolus Type 2): a phase 3, randomised, open-label, treat-to-target non-inferiority trial. Lancet 379:1498-1507

8. Heller S, Buse J, Fisher M et al (2012) Insulin degludec, an ultralongacting basal insulin, versus insulin glargine in basal-bolus treatment with mealtime insulin aspart in type 1 diabetes (BEGIN Basal-Bolus Type 1): a phase 3, randomised, open-label, treat-totarget non-inferiority trial. Lancet 379:1489-1497

9. Ratner RE, Gough SCL, Mathieu C et al (2013) Hypoglycaemia risk with insulin degludec compared with insulin glargine in type 2 and type 1 diabetes: a pre-planned meta-analysis of phase 3 trials. Diabetes Obes Metab 15:175-184

10. International Conference on Harmonisation of Technical Requirements for Registration of Pharmaceuticals for Human Use (2001) ICH harmonised tripartite guideline: guideline for good clinical practice. J Postgrad Med 47:45-50

11. World Medical Association (2009) Declaration of Helsinki. Ethical principles for medical research involving human subjects. J Indian Med Assoc 107:403-405

12. McAulay V, Deary I, Frier BM (2001) Symptoms of hypoglycaemia in people with diabetes. Diabet Med 18:690-705

13. Heller S (1999) Diabetic hypoglycaemia. Baillière's Best Pract Res Clin Endocrinol Metab 13:279-294

14. Deary IJ (2007) Symptoms of hypoglycaemia and effects on mental performance and emotions. In: Frier BM, Fisher M (eds) Hypoglycaemia in clinical diabetes, 2nd edn. Wiley, Chichester. doi:10.1002/9780470516270.ch2

15. Bowie CR, Harvey PD (2006) Administration and interpretation of the Trail Making Test. Nat Protoc 1:2277-2281

16. Reitan RM, Davison LA (1974) Clinical neuropsychology: current status and applications. V. H. Winston \& Sons, Washington, DC

17. Wechsler D (1981) WAIS-R manual: Wechsler adult intelligence scale-revised. Psychological Cooperation, New York

18. Wilkinson RT, Houghton D (1975) Portable four-choice reaction time test with magnetic tape memory. Behav Res Methods Instrum 7: $441-446$

19. Smeeton F, Shojaee-Moradie F, Jones RH et al (2009) Differential effects of insulin detemir and neutral protamine Hagedorn (NPH) insulin on hepatic glucose production and peripheral glucose uptake during hypoglycaemia in type 1 diabetes. Diabetologia 52:23172323

20. Tschritter O, Schäfer SA, Klett J et al (2009) Insulin detemir causes increased symptom awareness during hypoglycaemia compared to human insulin. Diabetes Obes Metab 11:1017-1026

21. Galassetti P, Davis SN (2000) Effects of insulin per se on neuroendocrine and metabolic counter-regulatory responses to hypoglycaemia. Clin Sci 99:351-362

22. Rossetti P, Porcellati F, Ricci NB et al (2008) Different brain responses to hypoglycemia induced by equipotent doses of the long-acting insulin analog detemir and human regular insulin in humans. Diabetes 57:746-756

23. Frier BM (2001) Hypoglycaemia and cognitive function in diabetes. Int J Clin Pract (S123):30-37

24. Dagogo-Jack S, Askari H, Morrill B et al (2000) Physiological responses during hypoglycaemia induced by regular human insulin or a novel human analogue, insulin glargine. Diabetes Obes Metab 2: $373-383$ 
25. Frier BM, Ewing FM, Lindholm A, Hylleberg B, Kanc K (2000) Symptomatic and counterregulatory hormonal responses to acute hypoglycaemia induced by insulin aspart and soluble human insulin in type 1 diabetes. Diabetes Metab Res Rev 16:262-268

26. Davis SN, Shavers C, Costa F, Mosqueda-Garcia R (1996) Role of cortisol in the pathogenesis of deficient counterregulation after antecedent hypoglycemia in normal humans. J Clin Invest 98:680-691

27. Raju B, McGregor VP, Cryer PE (2003) Cortisol elevations comparable to those that occur during hypoglycemia do not cause hypoglycemia-associated autonomic failure. Diabetes 52:2083-2089

28. Goldberg PA, Weiss R, McCrimmon RJ et al (2006) Autonomic failure. Diabetes 55:1121-1126

29. Ratner RE (2003) Insulin glargine versus NPH insulin in patients with type 1 diabetes. Drugs Today 39:867-876
30. Fanelli CG, Pampanelli S, Epifano L et al (1994) Long-term recovery from unawareness, deficient counterregulation and lack of cognitive dysfunction during hypoglycaemia, following institution of rational, intensive insulin therapy in IDDM. Diabetologia 37:12651276

31. Birkeland KI, Home PD, Wendisch U et al (2011) Insulin degludec in type 1 diabetes: a randomized controlled trial of a new-generation ultra-long-acting insulin compared with insulin glargine. Diabetes Care 34:661-665

32. Zinman B, Fulcher G, Rao PV et al (2011) Insulin degludec, an ultra-long-acting basal insulin, once a day or three times a week versus insulin glargine once a day in patients with type 2 diabetes: a 16-week, randomised, open-label, phase 2 trial. Lancet 377:924-931 